\title{
The role of surface elasticity in giant corrugations observed by scanning tunneling microscopes
}

\author{
W.A. Hofer ${ }^{\mathrm{a}, *}$, A. Garcia-Lekue ${ }^{\mathrm{a}}, \mathrm{H}$. Brune ${ }^{\mathrm{b}}$ \\ a Department of Chemistry, Surface Science Research Centre, The University of Liverpool, Liverpool L69 3BX, UK \\ ${ }^{\mathrm{b}}$ Faculté des Sciences de Base, Institut de Physique des Nanostructures, École Polytechnique Fédérale de Lausanne, \\ PHB - Ecublens, CH-1015 Lausanne, Switzerland \\ Received 11 July 2004; in final form 24 August 2004 \\ Available online 25 September 2004
}

\begin{abstract}
Due to the importance of scanning tunneling microscopy for atomic scale research the anomalously high corrugation values on close packed metal surfaces have been the subject of debate and extensive theoretical work in the past two decades. To date it remained unclear, how surface properties and electronic structures are related to forces and interactions. Here we show that elasticity, in particular the high elasticity of aluminum surfaces, enhances corrugation by up to one order of magnitude. The parameterfree simulations yield correct results for all close packed metal surfaces and emphasize the importance of atomic relaxations for chemisorption processes.
\end{abstract}

(C) 2004 Elsevier B.V. All rights reserved.

It is an astonishing fact that every second paper on surface chemistry or physics today is based on scanning tunneling microscopy (STM) experiments. The versatility of STM mostly derives from its ability to probe into most of the surface properties at the atomic scale. It has been shown conclusively, that it cannot only resolve atomic positions, but also their chemical nature [1], their chemical environment [2], magnetization [3], and even two dimensional surface states [4]. By contrast, STM theory has long been focused on electronic surface properties alone [5]. The method, even though it is generally reliable, involves as free parameter the distance between the two surfaces, which can be used to adjust simulated density contours to constant current linescans. In the estimates the actual distance between the atomic cores of surface and tip atoms varies widely from less than 300 to more than $1000 \mathrm{pm}[6,7]$. This distance range covers more than seven orders of magnitude in the tunnel-

\footnotetext{
${ }^{*}$ Corresponding author. Fax: +44 1517080662.

E-mail address: whofer@liverpool.ac.uk (W.A. Hofer).
}

ing current. In addition, the method breaks down completely on close packed metal surfaces, where measured corrugations exceed the values obtained from constant density contours by up to one order of magnitude [8-10].

Theoretically, this puzzle has been the focus of attention for more than 15 years. It is easy to see, why: if simulations of experiments in a simple case, like flat metal surfaces, leave up to $90 \%$ of the measured values unaccounted for, then interpretations of more subtle experiments are potentially imprecise by the same amount. It deprives theoretical work in this field of a sound scientific basis. This basis can only come from a detailed understanding of the physical processes involved in the imaging process. Two separate models have been put forward to account for the deviations: it was either thought to be due to electronic effects, or due to the interactions between atoms at opposite sides of the tunneling junction. The first model has been favored in the work of Chen [11], where it was thought that states of $\mathrm{d}_{z^{2}}$ symmetry at the STM tip lead to an 
enhancement of corrugation. The assumption is backed to some extent by electronic structure calculations of Tsukada et al. [12], who showed that a tungsten cluster displays a state of $\mathrm{d}_{z^{2}}$ symmetry at the Fermi level. The same line of reasoning was used in a paper by Jacobsen et al. [13] in 1995. However, as Sacks [14] reported recently, the obtained corrugation with these states is still one order of magnitude below experimental values. Along a different line of research Doyen placed the emphasis on the tunneling process itself, accounting for increased corrugation by solving the scattering problem with a modified Dyson equation, obtaining corrugation enhancements of the right magnitude [15]. All methods based on an enhancement due to electronic structure are implicitly based on the assumption, that only very few electron transitions between surface and tip are responsible for the observed corrugation values. This assumption, however, is contradicted by explicit calculations within the Bardeen method [16], where typically a few hundred tunneling channels are obtained for bias voltages of $50-100 \mathrm{mV}$. Therefore, the theoretical understanding of the problem tipped ultimately in favor of interactions between surface and tip atoms. Here, the problem of dynamic processes in STM scans was until very recently treated by semi empirical methods. Pair potentials were used by Soler et al. [17] and by Clarke et al. [18] to account for corrugation enhancements on graphite and copper surfaces, respectively. In this case it proved difficult to relate current values in the experiments, which are a measure for the distance between the two surfaces, to the forces and relaxations of atoms, since pair-potentials decay very rapidly beyond $300 \mathrm{pm}$ : a detailed analysis of the interplay between interactions and tunneling currents remained elusive also with this method. The solution, for gold surfaces, was presented by Hofer et al. [19]; it involved calculating the forces and relaxations of coupled systems, and to determine the effect on constant current contours within a firstprinciples approach. It was also shown that tunneling current and interaction energy are in fact proportional to each other [20]. This result, contradicting earlier assumptions by Chen [21], is confirmed by experimental data [22]. However, it remained unknown so far, whether the same physical process applies to the case of aluminum surfaces, and equally, how the difference between the two surfaces can be accounted for. Here, we provide the solution to this problem and complete the analysis of tunneling currents, interactions, and relaxations by presenting a first principles method for computing dynamic constant current contours in the simulation of STM experiments. Our simulation results for $\mathrm{Al}\left(\begin{array}{lll}1 & 1 & 1\end{array}\right)$ provide the first quantitative agreement with the experimental corrugation amplitudes for realistic tip-sample distances. Also the experimentally observed differences between the apparent heights of fcc and hcp hollow sites are reproduced.
We have calculated the tunneling currents on the three close packed metal surfaces $\mathrm{Cu}\left(\begin{array}{lll}1 & 1 & 1\end{array}\right), \mathrm{Au}\left(\begin{array}{lll}1 & 1 & 1\end{array}\right)$, and $\mathrm{Al}\left(\begin{array}{lll}1 & 1 & 1\end{array}\right)$ in a two step procedure. The electronic structure of surfaces and tip has been calculated by standard density functional theory, the Vienna ab initio Simulation Program (VASP) [23,24]. For the description of the ionic cores we used the Projector Augmented Waves (PAW) method in all calculations [25], the exchange-correlation potential was described following Perdew et al. [26]. The surface was mimicked by a 13layer film, the vacuum range above the surface was about $1500 \mathrm{pm}$. This guarantees a smooth decay of the surface wavefunctions into the vacuum region. For the integration of the surface Brillouin zone we employed a mesh of 42 special $k$-points. The tip was mimicked by a tungsten pyramid of seven layers in $\left(\begin{array}{lll}1 & 1 & 0\end{array}\right)$ orientation, the tip apex was atomically sharp (single atom apex). The setup of the tip calculation, the method, and the vacuum range were the same as for the surface. The tip structure was mimicked by a three layer tungsten film with a two layer pyramid on either side (see Fig. 1). The apex consists of a single atom. The atoms of the pyramid were changed from tungsten to aluminum in one case $\left(\mathrm{Al}\left(\begin{array}{lll}1 & 1 & 1\end{array}\right)\right)$ to study the effects of tip contamination. Here, we used a mesh of 30 special $k$-points for the Brillouin zone integration. The current was determined from the electronic structure of the fully relaxed 13-layer surface film and a 7-layer tip by integrating the contributions of all states within a given energy interval, determined by the bias voltage. The numerical procedure to obtain the tunneling current is described elsewhere [16]. In general, we found that with a tungsten pyramid in $\left(\begin{array}{lll}1 & 1 & 0\end{array}\right)$ orientation the corrugation is very small and in the range of a few pm. This is consistent with simulations performed on other surfaces, where it was found that tungsten at the tip apex in most cases reduces the corrugation substantially, compared to an apex atom of, e.g., the same chemical nature as surface atoms [16]. We then introduced interaction forces and the induced relaxations in the following way. Assuming that the interaction energy $E$ is given by $E=-\alpha G$, where $G$ is the tunneling conductance and $\alpha$ a constant, which is determined by first principles simulations [20], then the vertical relaxation of surface atoms $z$ can be determined within the harmonic approximation by $E=$ $-k z^{2}$. Given that the tunneling current at one point of the surface follows an exponential decay $I(z)=I_{0} \mathrm{e}^{-\kappa z}$, it is possible to relate the modified current $I^{\prime}(z)$, due to relaxations of the surface atoms, to the conductance at this very same point with

$I^{\prime}(z)=I(z) \exp \kappa \sqrt{\frac{P \alpha G}{k}}$.

The projection $P$ accounts for the fact that atomic motion at the surface is restricted to the vertical 


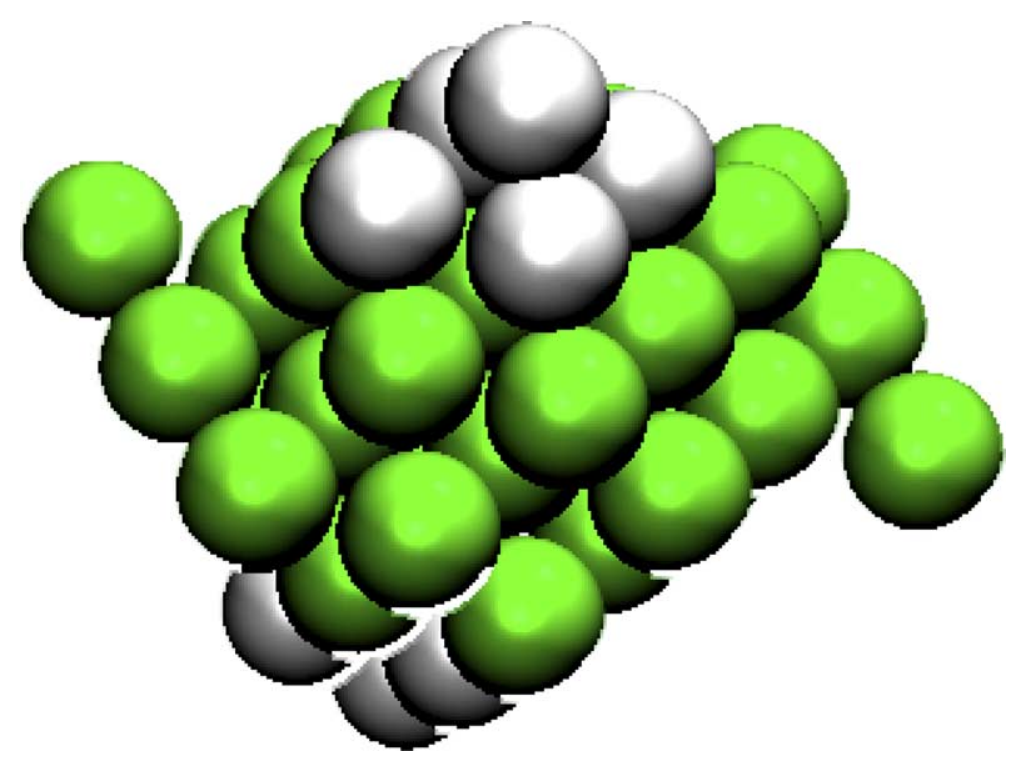

Fig. 1. Tip model in the calculation. The tip is simulated by a three layer tungsten film (dark green atoms), with a pyramid of two layers on either side (light atoms). The chemical nature of the pyramid atoms reflects clean or contaminated tips. In most cases we used tungsten, in case of $\mathrm{Al}(111)$ constant current contours were also simulated with $\mathrm{Al}$ atoms. (For interpretation of the references to color in this figure legend, the reader is referred to the web version of this article.)

direction; near the position of a surface atom $P(d=0)=1 . d$ is the lateral distance of the STM tip from the nearest surface atom. Near the threefold hollow site of the surface it is close to $1 / 3$, as the interaction energy will then be equally distributed between three adjacent atoms. In simulations an additional requirement is numerical stability, since current contours are very sensitive to discontinuous changes of the derivative with respect to $d$. We have therefore set

$P(d)=\cos \left(\frac{z}{\sqrt{z^{2}+d^{2}}}\right) \cdot a\left[1-b \tanh \left(\frac{4 d}{d_{0}}-2\right)\right]$.

The parameters $a$ and $b$ depend on the symmetry of the surface, $d_{0}$ is the distance between the hollow site and the on top site. In principle, all constants in this equation can be calculated from first principles. However, since it was observed that the constant $\alpha$ increases close to exponentially with the combined Wigner-Seitz radii of surface and tip atoms, we fitted it to the radii based on two explicit calculations for the (1 111$)$ surface of gold and the $\left(\begin{array}{lll}1 & 0 & 0\end{array}\right)$ surface of copper [20]. The decay $\kappa$ of the current at a particular position was obtained by a least-square fit, the numerical routines reported only negligible (less than $2 \%$ ) variations from an exponential decay. The elastic constant $k$ of surface atoms was obtained by a standard procedure: surface atoms were moved outward by about $4 \mathrm{pm}$ and the electronic structure fully relaxed. The forces on the atom can then directly be obtained from our density functional method $[23,24]$. Here, we found that all noble metals have a comparable elasticity, the values for $\mathrm{Cu}(k=3.77 \mathrm{eV} /$ $\left.\AA^{2}\right), \mathrm{Ag}\left(k=2.96 \mathrm{eV} / \AA^{2}\right)$ and $\mathrm{Au}\left(k=3.22 \mathrm{eV} / \AA^{2}\right)$ differ by less than $30 \%$. However, the corresponding value for $\mathrm{Al}\left(k=0.89 \mathrm{eV} / \AA^{2}\right)$ is substantially smaller, indicating a much higher elasticity of the $\mathrm{Al}\left(\begin{array}{lll}1 & 1 & 1\end{array}\right)$ surface. The harmonic constants have originally been calculated for setups comprising a single unit cell. As this amounts to a displacement of the whole surface layer, we performed additional calculations for aluminum, using a $3 \times 3$ unit cell and a film of seven layers. We find a slightly higher value for $k$. However, the variation to the value used in the calculations is less than $20 \%$. Given the exponential factor of Eq. 1 this amounts to a slight deviation of less than $10 \%$ for the enhancement of tunneling currents due to relaxations. For reasons of consistency all relaxations have been calculated with the values obtained for single unit cells. In addition, we estimated the harmonic constant in case of a coupled system like the one used in [27]. In this case we obtain a substantially larger value, which we attribute to the much lower screening of nuclear charge on $\mathrm{Al}$ compared to e.g., $\mathrm{Cu}$ and $\mathrm{Au}$. This may also explain, to some extent, why the range of relaxations in a coupled calculation is much lower $(22 \mathrm{pm}$ [27]) than is necessary for an explanation of giant corrugation measurements on $\mathrm{Al}\left(\begin{array}{lll}1 & 1 & 1\end{array}\right)$. Following the procedure described above the ensuing current includes the relaxations of surface atoms, and it describes the dynamic constant current contour from first principles.

In order to illustrate the contribution of elastic effects we have calculated, in first instance, the corrugation on $\mathrm{Cu}\left(\begin{array}{lll}1 & 1 & 1\end{array}\right)$ based on the electronic structure alone. This calculation leads to a slightly anticorrugated surface. Such a result has, to our knowledge, never been observed experimentally. Fig. 2 displays three current 


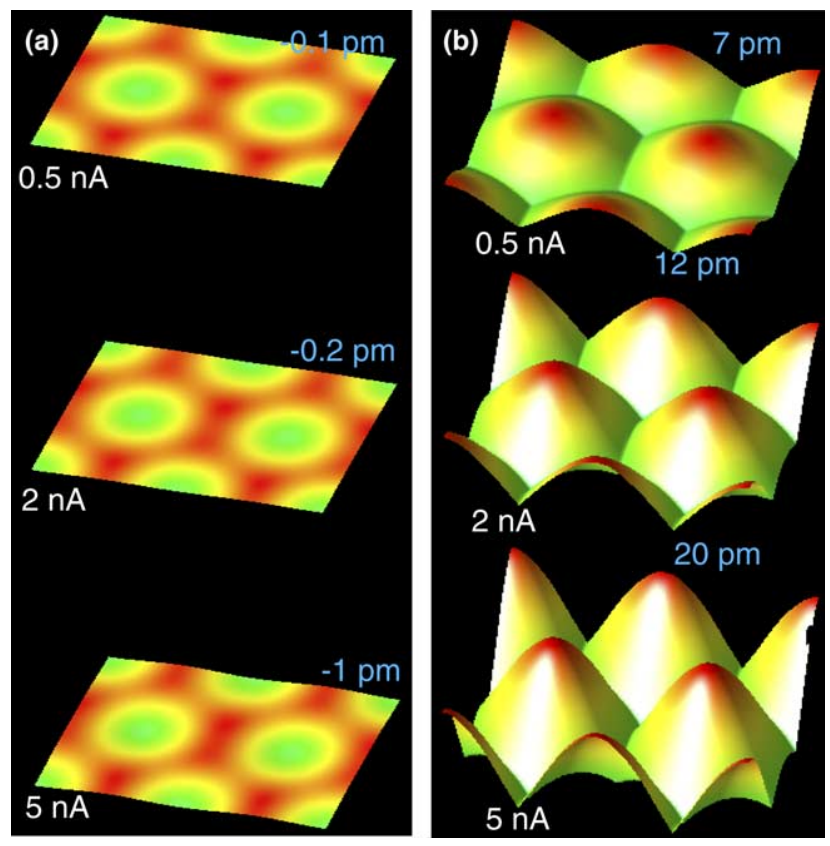

Fig. 2. Constant current contours on $\mathrm{Cu}\left(\begin{array}{lll}1 & 1 & 1\end{array}\right)$ from the electronic structure alone (a), and including relaxations of the surface (b). Current values are given, the bias voltage in the simulations was -100 $\mathrm{mV}$. While the surface would appear anticorrugated (atoms are seen as holes) from the electronic structure alone (a), the relaxation of surface atoms leads to positive corrugation over the whole current range (b). (For interpretation of the references to color in this figure legend, the reader is referred to the web version of this article.)

contours on $\mathrm{Cu}\left(\begin{array}{lll}1 & 1 & 1\end{array}\right)$ based on the electronic structure alone (a), and three dynamic contours including forces and relaxations (b). We conclude from this result, that also simulations including the tip can be misleading, if they do not account for the relaxation of surface atoms. As revealed by the dynamic contours, the effect of interactions is that the surface is now positively corrugated over the whole distance range. We obtain similar corrugation enhancements as on the $\mathrm{Au}\left(\begin{array}{lll}1 & 1 & 1\end{array}\right)$ surface [19], where we find a corrugation value of $24 \mathrm{pm}$ under tunneling conditions of $-100 \mathrm{mV} / 5 \mathrm{nA}$. The result is in good agreement with experiments $[8,9]$ and with our previous calculations, based on only two points of the contour map [19]. We also observe an increase of the apparent barrier height as the tip approaches the surfaces, as reported experimentally [28]. Due to the rapid decay of chemical bonding substantial interaction effects are limited to the distance range below $600 \mathrm{pm}$.

On $\mathrm{Al}\left(\begin{array}{lll}1 & 1 & 1\end{array}\right)$ we observe a dramatic increase of the corrugation amplitude due to dynamic effects. To estimate the effect of Al possibly adsorbed at the STM tip, we have performed three separate simulations, using: (i) a tungsten tip in (1 110$)$ orientation with a tungsten pyramid; (ii) a tungsten tip with an Al pyramid; (iii) an aluminum tip in ( 1110$)$ orientation with an $\mathrm{Al}$ pyramid. To compare with experiments [10] the bias voltage was set to -50 and $-20 \mathrm{mV}$, respectively. The increase of dynamic effects on this surface is partly due to the higher current observed on this surface (the maximum current before dynamic adjustments is about 12 $\mathrm{nA}$, while it is only $5 \mathrm{nA}$ on the other surfaces). This is due to the lower workfunction of $\mathrm{Al}$ compared to $\mathrm{Au}$ or $\mathrm{Cu}$. However, this feature mainly shifts the distance scale, at which dynamic effects are observed. The main reason for the increase is thus the much higher elasticity of the aluminum surface. Considering the enhancement factor in Eq. 1, an increase of the current value by 2 and a decrease of the elastic constant $k$ by 4 will yield a corrugation enhancement of 2.82. Even if the exponential decay is somewhat smaller than, e.g., on $\mathrm{Au}\left(\begin{array}{ll}1 & 1\end{array}\right)$, the increase is still substantial. As shown in Fig. 3, this leads to a surface corrugation of up to 70 pm $(-50 \mathrm{mV}$, Fig. 3c). We obtain similar results for both tungsten tips (with a tungsten or aluminum pyramid, respectively), but only about half the corrugation value for the aluminum tip. These results agree well with experiments, if we consider that the low corrugation values with clean tungsten tips were mainly due to the large tip radius of more than $50 \mathrm{~nm}$. The tip in the high corrugation experiments was therefore atomically sharp and covered by comparatively few $\mathrm{Al}$ atoms. The experimental corrugation amplitudes reported in [10] have remained a puzzle for more than 15 years. Here, we find the solution of this puzzle: as for $\mathrm{Al}$ the surface atoms are less strongly bound to the surface than for noble metals, their outward relaxation under tunneling conditions is very large. Combined with the changes of forces, as the tip moves from the on-top to the hollow position, this gives rise to unexpected corrugation values. The main increase of corrugation occurs between 1 and 10 $\mathrm{M} \Omega$ tunneling resistance, defined as the ratio of bias potential and tunneling current. In this range the corrugation increases from 20 to $70 \mathrm{pm}$, the tunneling resistance decreases faster than exponential (Fig. 3c). To analyze the stability in the limit of high corrugations we also computed the interaction energy in this situation. The value we obtain is less than $0.5 \mathrm{eV}$, corresponding to a an absolute displacement of $\mathrm{Al}$ atoms by $73 \mathrm{pm}$ as the tip is in the on-top position. Comparing with our previous first principles calculations [19], these values are lower than the energy threshold of about $1 \mathrm{eV}$ for the jump into contact and also substantially lower than the displacement of more than $130 \mathrm{pm}$ related to it. In order to verify that the simulated current and displacement values remain within the elastic regime we performed a separate calculation of a coupled $\mathrm{Al}\left(\begin{array}{lll}1 & 1 & 1\end{array}\right)$ surface and Al tip, in a geometry also used by another theory group [27]. The density functional calculation was performed with projector augmented-waves, the energy cutoff was set to $240 \mathrm{eV}$. We find in this case that the jump into contact occurs at an interaction energy of $0.7 \mathrm{eV}$, a result confirmed by Blanco et al.[27] (see Fig. 3). The jump into contact is very sudden, within a 

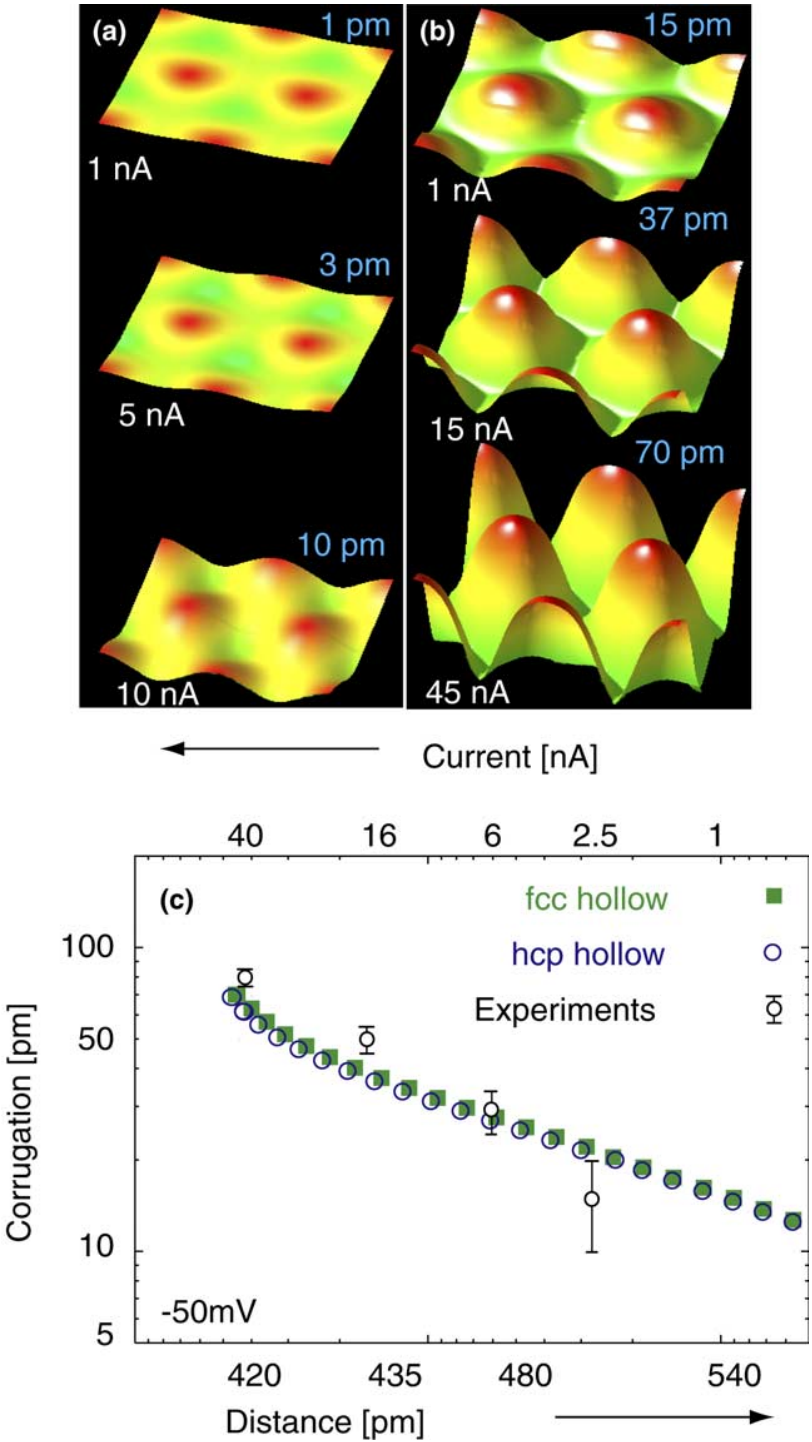

Fig. 3. Constant current contours on $\mathrm{Al}\left(\begin{array}{lll}1 & 1 & 1\end{array}\right)$ for a bias voltage of $-50 \mathrm{mV}$. Even though the surface appears already corrugated in the contours based on the electronic structure (a), interactions lead to very large enhancements and quite singular corrugations in excess of $70 \mathrm{pm}$ (b). The apparent height of surface atoms increases mainly in the range from 10-1 $\mathrm{M} \Omega$ tunneling resistance, its maximum value in the simulation is about $80 \mathrm{pm}$ (c). (For interpretation of the references to color in this figure legend, the reader is referred to the web version of this article.)

distance variation of less than $0.01 \AA$. We attribute this sudden onset to the somewhat artificial setup, which leads to substantially lower elasticity as observed on isolated surfaces. In contrast to the results by Blanco et al. we find a distance of $3.9 \AA$ for this effect, calculating the relaxations with a local resolution of $0.05 \AA$ from 4.0 to $3.8 \AA$ and in steps of $0.01 \AA$ near $3.9 \AA$. We attribute the difference to the fact that the calculation of Blanco et al. is limited to only one point in the range from 4.25 to $3.75 \AA$. Given the energy and the distance range in our simulation it is thus safe to conclude that it is well within the elastic range.
The most startling result of STM experiments on $\mathrm{Al}\left(\begin{array}{lll}1 & 1 & 1\end{array}\right)$ is the ability to resolve the position of subsurface atoms. Atoms in the subsurface layer are found at the hcp hollow site, while the position at the fcc hollow site is empty. Upon decreasing the bias voltage to -20 $\mathrm{mV}$ at a tunnel current of $40 \mathrm{nA}$ (corresponding to a conductance of $2 \mathrm{M}^{-1}$ ) the simulations show a difference in apparent height between the two three-fold hollow sites of about $5 \mathrm{pm}$, which is in excellent agreement with the difference measured in experiment $(5 \mathrm{pm}$, see Fig. 4).

This mechanism is also thought to account for the giant corrugations observed on graphite surfaces $[17,29]$. In this case, the graphite layers are only weakly bound, and the elasticity of individual atoms will be sub-

(a)

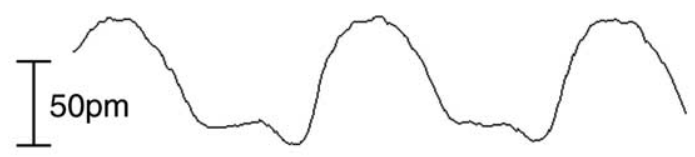

(b)
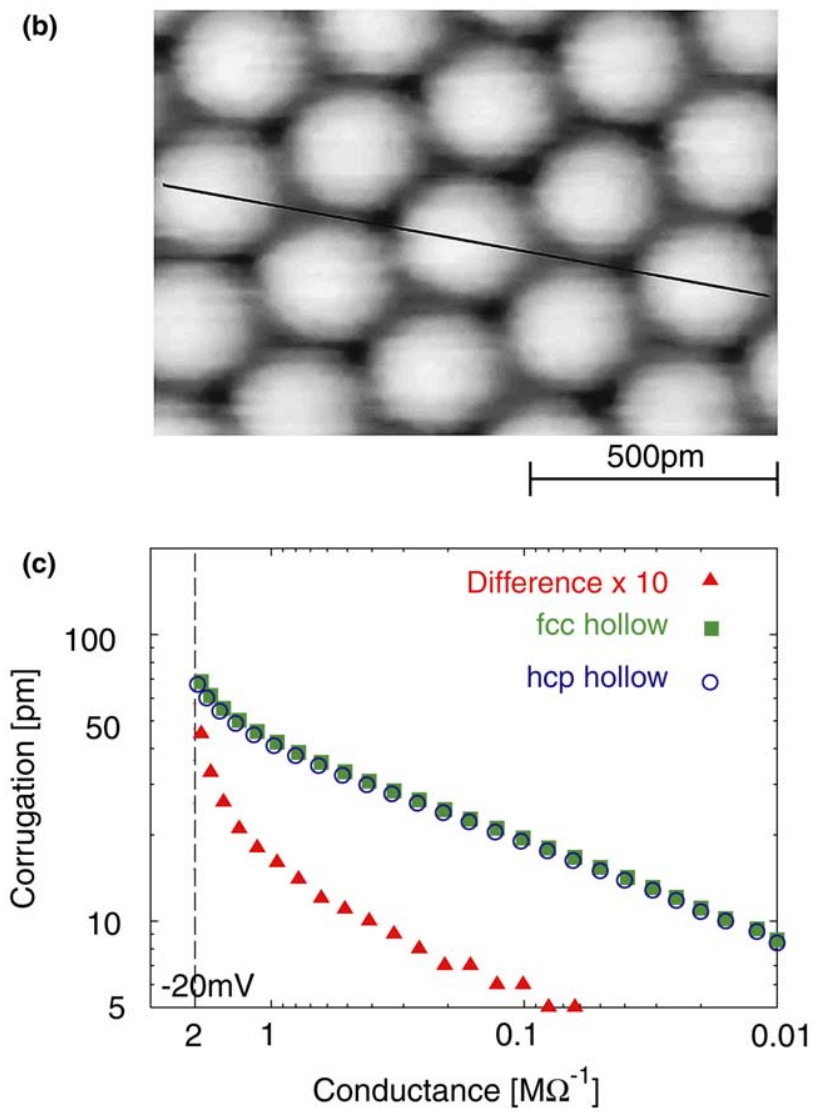

Fig. 4. Resolution of the position of subsurface atoms. The atoms of the subsurface layer are located at the hcp hollow site, which shows a slightly higher contour (about $5 \mathrm{pm}$ ) than the fcc hollow sites, where subsurface atoms are missing. (a) Experimental constant current profile along the $\left[\begin{array}{lll}1 & 1 & \overline{2}\end{array}\right]$ direction of the $\mathrm{Al}\left(\begin{array}{lll}1 & 1 & 1\end{array}\right)$ surface including hcp and fcc hollow sites. (b) Experimental constant current contour. (c) simulated corrugation values and difference between hcp and fcc hollow sites. (For interpretation of the references to color in this figure legend, the reader is referred to the web version of this article.) 
stantially higher than, e.g., for $\mathrm{Al}\left(\begin{array}{lll}1 & 1 & 1\end{array}\right)$. This entails that the dynamic part of surface corrugation will well surpass the values reported here for $\operatorname{Al}\left(\begin{array}{llll}1 & 1 & 1\end{array}\right)$. From the vertical range of dynamic changes on elastic surfaces and the obvious ubiquity of forces in scanning probe experiments we also conclude that relaxations will play a major role in every case, where surface corrugation is higher than its estimate from the surface electronic structure. If scanning probe experiments are to probe into subtle surface effects, including the electronic structure of surface and tip and dynamic aspects of the experiments seems thus necessary.

\section{Acknowledgements}

W.A.H. thanks the Royal Society for the award of a University Research Fellowship, A.G.L. is supported by a Marie-Curie Fellowship of the European Commission. H.B. thanks J. Wintterlin, R. Behm, and G. Ertl for permission of publication of Fig. 4.

\section{References}

[1] P. Varga, M. Schmid, Appl. Surf. Sci. 141 (1999) 287.

[2] Y. Gauthier, M. Schmid, S. Padovani, E. Lundgren, V. Bu, G. Kresse, J. Redinger, P. Varga, Phys. Rev. Lett. 87 (2001) 036103.

[3] M. Bode, S. Heinze, A. Kubetzka, O. Pietzsch, X. Nie, G. Bihlmayer, S. Blügel, R. Wiesendanger, Phys. Rev. Lett. 89 (2002) 237205.

[4] J. Kliewer, R. Berndt, E.V. Chulkov, V.M. Silkin, P.M. Echenique, S. Crampin, Science 288 (2000) 1399.

[5] J. Tersoff, D.R. Hamann, Phys. Rev. B 31 (1985) 805.

[6] M.V. Bollinger, J.V. Lauritsen, K.W. Jacobsen, J.K. Nørskov, S. Helveg, F. Besenbacher, Phys. Rev. Lett. 87 (2001) 196803.
[7] M. Bode, S. Heinze, A. Kubetzka, O. Pietzsch, X. Nie, G. Bihlmayer, S. Blügel, R. Wiesendanger, Phys. Rev. Lett. 89 (2002) 237205.

[8] V.M. Hallmark, S. Chiang, J.F. Rabolt, J.D. Swalen, R.J. Wilson, Phys. Rev. Lett. 59 (1987) 2879.

[9] J.V. Barth, H. Brune, G. Ertl, R.J. Behm, Phys. Rev. B 42 (1990) 9307.

[10] J. Wintterlin, J. Wiechers, H. Brune, T. Gritsch, H. Höfer, R.J. Behm, Phys. Rev. Lett. 62 (1989) 59.

[11] C.J. Chen, Phys. Rev. Lett. 65 (1990) 448.

[12] M. Tsukada, K. Kobayashi, N. Isshiki, H. Kageshima, Surf. Sci. Rep. 13 (1991) 265.

[13] J. Jacobsen, B. Hammer, K.W. Jacobsen, J.K. Nørskov, Phys. Rev. B 52 (1995) 14954.

[14] W. Sacks, Phys. Rev. B 61 (2000) 7656.

[15] G. Doyen, D. Drakova, M. Scheffler, Phys. Rev. B 47 (1993) 9778.

[16] W.A. Hofer, A.S. Foster, A.L. Shluger, Rev. Mod. Phys. 75 (2003) 1287.

[17] J.M. Soler, A.M. Baro, N. Garcia, H. Rohrer, Phys. Rev. Lett. 57 (1986) 444.

[18] A.R.H. Clarke, J.B. Pethica, J.A. Nieminen, F. Besenbacher, E. Laegsgaard, I. Stensgaard, Phys. Rev. Lett. 76 (1996) 1276.

[19] W.A. Hofer, A.J. Fisher, R.A. Wolkow, P. Grütter, Phys. Rev. Lett. 87 (2001) 236104.

[20] W.A. Hofer, A.J. Fisher, Phys. Rev. Lett. 91 (2003) 036803.

[21] C.J. Chen, Introduction to Scanning Tunneling Microscopy, Oxford University Press, Oxford, 1993.

[22] A. Schirmeisen, G. Cross, A. Stalder, P. Grütter, U. Dürig, New J. Phys. 2 (2000) 29.

[23] G. Kresse, J. Hafner, Phys. Rev. B 47 (1993) 558.

[24] G. Kresse, J. Furthmüller, Phys. Rev. B 54 (1996) 11169.

[25] P.E. Blöchl, Phys. Rev. B 50 (1994) 17953.

[26] J.P. Perdew, J.A. Chevary, S.H. Vosko, K.A. Jackson, M.R. Pederson, D.J. Singh, C. Fiolhais, Phys. Rev. B 46 (1991) 6671.

[27] After submission of the paper we became aware of a simulation on $\mathrm{Al}\left(\begin{array}{lll}1 & 1 & 1\end{array}\right)$ by a group in Madrid: J.M. Blanco, C. Gonzalez, P. Jelnek, J. Ortega, F. Flores, R. Perez, Phys. Rev. B 70 (2004) 085405 .

[28] J.K. Gimzewski, R. Möller, Phys. Rev. B 36 (1987) 1284.

[29] S. Hembacher, F.J. Giessibl, J. Mannhart, C.F. Quate, Proc. Natl. Acad. Sci. 100 (2003) 12539. 